\title{
Una de cada cinco muertes en el mundo se asocian a una alimentación no saludable: ¿Cuál es la realidad chilena?
}

\section{One in five deaths are associated with an unhealthy diet worldwide: what is the current scenario in Chile?}

Las enfermedades no transmisibles (ENT) causan 41 millones de muertes cada año, $(71 \%$ del total de muertes en el mundo); siendo la mayoría de las muertes causadas por enfermedades cardiovasculares (ECV) (17,9 millones), cáncer (9 millones) y diabetes mellitus tipo 2 (DMT2) (1,6 millones) ${ }^{1}$. La alimentación no saludable es uno de los principales factores de riesgo que ha contribuido al incremento en la prevalencia y mortalidad asociada a $\mathrm{ENT}^{2}$.

La revista "The Lancet" publicó los resultados del estudio "Global Burden of Disease" (GBD 2017) $)^{3}$ el cual verifica el impacto de una alimentación no saludable en el riesgo de desarrollar ENT y mortalidad asociada. Este estudio analizó las tendencias de consumo de 15 factores de riesgo relacionados con la dieta, en 195 países entre los años 1990 y 2017; encontrándose que éstos fueron responsables de 11 millones de muertes y 255 millones de DALYs (Años de Vida Ajustados por Discapacidad). Los principales factores de riesgo responsables del $50 \%$ de las muertes y $66 \%$ de DALYs a nivel mundial, fueron la alta ingesta de sodio, bajo consumo de granos enteros y bajo consumo de frutas (Figura 1). El consumo de alimentos y nutrientes saludables fue subóptimo, especialmente frutos secos, leche y granos enteros. Por el contrario, los no saludables, superaron los niveles óptimos de consumo, destacándose bebidas azucaradas, carnes procesadas y sodio. A nivel mundial, del total de muertes atribuibles a la dieta no saludable, 10 millones fueron causadas por ECV, seguido de cáncer (913.090 muertes) y DMT2 (338.714 muertes) ${ }^{3}$.

En relación a América del Sur, el GDB 2017 reportó que el impacto de la dieta subóptima sobre la tasa de mortalidad fue de 190 muertes/100.000 habitantes (18\% del total de muertes en adultos), siendo Perú el país en el cual los hábitos de alimentación causaron menos muertes (Figura 2). Al analizar la situación de Chile, la tasa de mortalidad asociada a una alimentación no saludable en el año 2017 fue de 158 muertes/100.000 habitantes (17\% del total de muertes registradas en el país); de éstas, por cada 100.000 habitantes, 120 muertes fueron atribuidas a ECV, 24 a cáncer y 10 a diabetes ${ }^{3}$.

Considerando el perfil de consumo de los principales factores dietéticos abordados en el GBD 2017, en Chile el
María Adela Martínez-Sanguinetti ${ }^{*}$, Ana María Leiva ${ }^{2}$, Fanny Petermann-Rocha ${ }^{3,4}$, Marcelo Villagrán ${ }^{5}$, Claudia Troncoso-Panto$j^{6}{ }^{6}$, Carlos Celis-Morales ${ }^{4,7}$, en representación del Grupo ELHOC (Epidemiology of Lifestyle and Health Outcomes in Chile).

1. Instituto de Farmacia, Facultad de Ciencias,
Universidad Austral de Chile, Valdivia, Chile.
Universidad Austral de Chile, Valdivia, Chile.
2. Instituto de Anatomía, Histologí y Patolói, Facultad de Medicia,
3. Institute of Health and Wellbeing, University of Glasgow,
Glasgow, United Kingdom.
4. BHF Glasgow Cardiovascular Research Centre,
Institute of Cardiovascular and Medical Science,
University of Glasgow, Glasgow, United Kingdom.
5. Departamento de Ciencias Básicas. Facultad de Medicina.
Universidad Católica de la Santísima Concepción, Concepción, Chile.
6niversidad Católica de la Santísima Concepción, Concepción, Chile.
7. Centro de Investigación en Fisiología del Ejercicio - CIFE,
Universidad Mayor. Santiago, Chile
*Dirigir correspondencia a: María Adela Martínez-Sanguinetti,
Instituto de Farmacia, Facultad de Ciencias,
Universidad Austral de Chile, Valdivia, Chile.
Teléfono: 56-632 293995.
Email: mmartin3@uach.cl

Este trabajo fue recibido el 07 de mayo de 2019. Aceptado con modificaciones: 13 de mayo de 2019. Aceptado para ser publicado: 05 de junio de 2019.

$98,4 \%$ de la población consume $>5 \mathrm{~g}$ sal/día, superando en más de $70 \%$ el nivel máximo de sodio permitido ${ }^{4}$. Esto evidencia que, a pesar de la estrategia nacional de reducción de sal en el pan y las campañas para disminuir el consumo de sal en las comidas, es necesario ampliar esta restricción a otros alimentos de uso masivo, que en conjunto con la Ley de Etiquetado de Alimentos contribuyan a disminuir el consumo de sodio en la población y así controlar el principal factor de riesgo dietético responsable del mayor porcentaje de muertes por esta causa ${ }^{5}$. En cuanto al consumo de alimentos protectores de ENT, la última Encuesta Nacional de Salud ${ }^{6}$, informó un bajo grado cumplimiento de las recomendaciones de las Guías Alimentarias en la población chilena ${ }^{7}$, destacándose un 
bajo consumo de frutas y verduras (15\%), de legumbres $(24,4 \%)$ y pescado $(9,2 \%)$.

Considerando los datos entregados por el GDB 2017 y las actuales conductas alimentarias de la población chilena, es necesario seguir reforzando todas las acciones orientadas a educar a la población respecto a los beneficios de la incorporación en la dieta de alimentos saludables, desde la infancia y a través de todo el ciclo vital.

Figura 1. Número de muertes por enfermedades cardiovasculares atribuibles a los 15 factores de riesgo asociados con la alimentación, a nivel mundial el 2017. Adaptación de GBD 2017 (3).

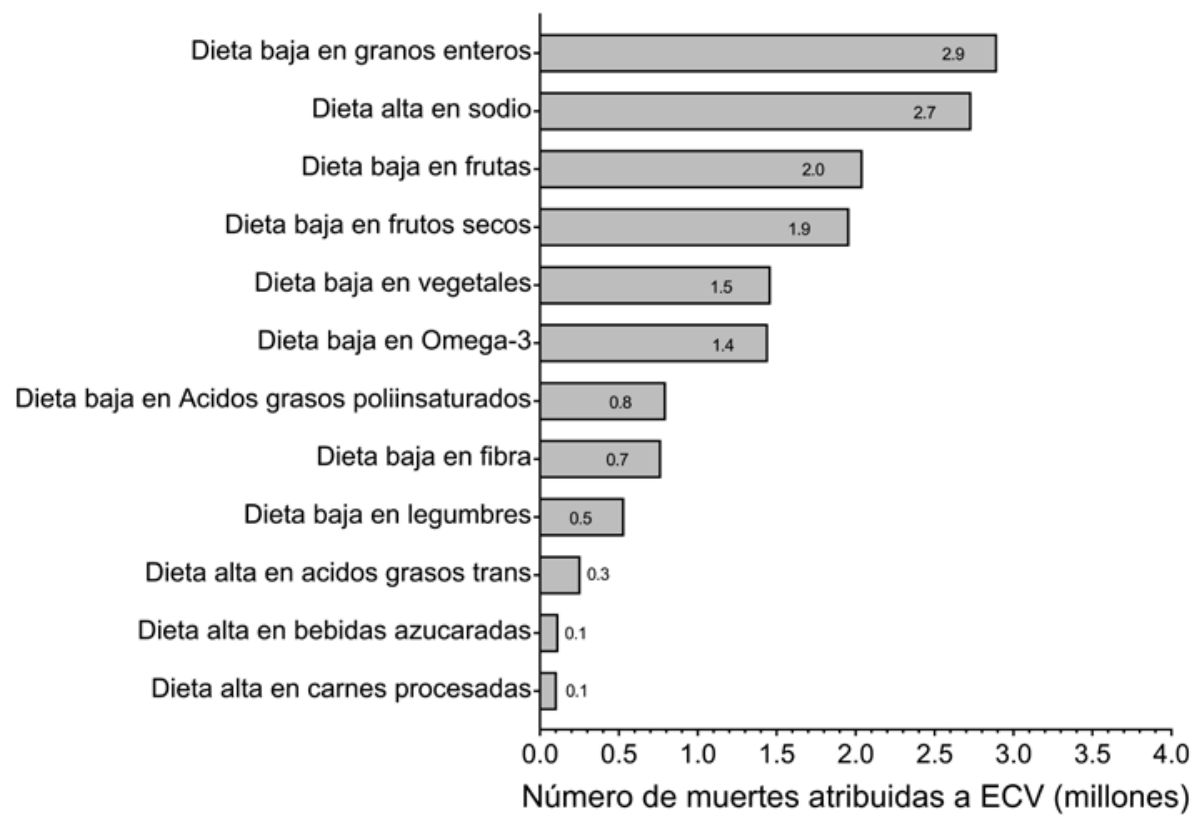

Figura 2. Porcentage de muertes (total y por causa especifica) atribuibles a no cumplir con las recomendaciones alimentarias. Adaptado de GBD 2017

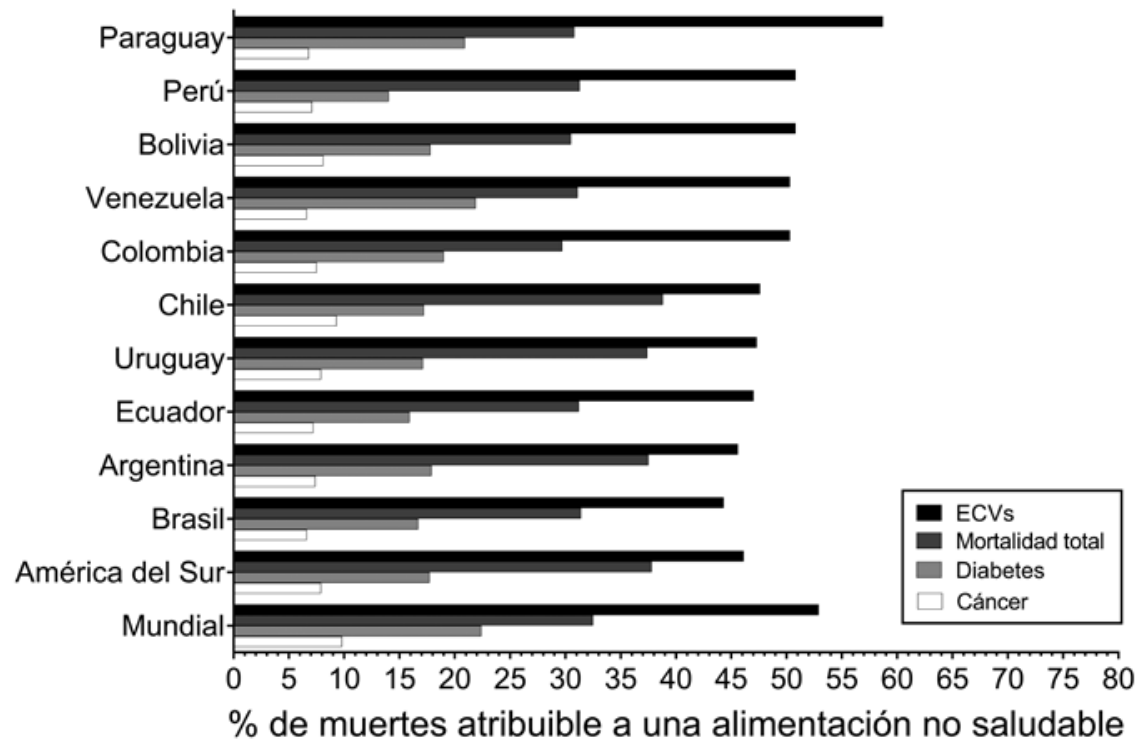




\section{BIBLIOGRAFIA}

1. WHO. Non-communicable Diseases. World Health Organization. June 1, 2018. https://www.who.int/es/newsroom/fact-sheets/detail/noncommunicable-diseases

2. GBD 2015 Risk Factors Collaborators. Global, regional, and national comparative risk assessment of 79 behavioural, environmental and occupational, and metabolic risks or clusters of risks, 1990-2015: a systematic analysis for the Global Burden of Disease Study 2015. Lancet 2016; 388(10053): 1659-1724.

3. GBD 2017 Diet Collaborators. Health effects of dietary risks in 195 countries, 1990-2017: a systematic analysis for the Global Burden of Disease Study 2017. The Lancet. Published online April 3, 2019. http://dx.doi.org/10.1016/ S0140-6736(19)30041-8

4. ENCA. National Survey of Food Consumption. Final report.
Department of Nutrition. School of Nutrition. School of Public Health. School of Medicine. Microdata Center, Faculty of Economics and Business. Universidad de Chile. https://www. minsal.cl/sites/default/files/ENCA-INFORME_FINAL.pdf

5. Law 20,606. On the nutritional composition of foods and their advertising. July 6, 2012. Library of the National Congress of Chile. Chilean legislation. https://www.minsal.cl/sites/default/ files/LEY-20606_06-JUL-2012.pdf

6. MINSAL. First and Second Results of the National Health Survey 2016-2017. Ministry of Health, Chile's goverment. http://epi.minsal.cl/resultados-encuestas/

7. MINSAL. Study to Review and Update the Dietary Guidelines for the Chilean Population. Final report. May 16, 2013. Ministry of Health. Chile's goverment.https://www.minsal. $\mathrm{cl} /$ portal/url/item/dde0bc471a56a001e040010165012224. pdf 